\title{
Confirmation of EP300 gene mutations as a rare cause of Rubinstein-Taybi syndrome
}

\author{
Nicole Zimmermann ${ }^{1}$, Ana Maria Bravo Ferrer Acosta ${ }^{2}$, Jürgen Kohlhase ${ }^{2}$ and \\ Oliver Bartsch*,1
}

${ }^{1}$ Institute for Human Genetics, Johannes Gutenberg-University, Mainz, Germany; ${ }^{2}$ Institute for Human Genetics and Anthropology, University of Freiburg, Freiburg, Germany

The Rubinstein-Taybi syndrome (RSTS, MIM 180849), a dominant Mendelian disorder with typical face, short stature, skeletal abnormalities, and mental retardation, is usually caused by heterozygous mutations of the CREBBP gene, but recently, EP300 gene mutations were reported in three individuals. Using quantitative PCR (for the CREBBP and EP300 genes) and genomic sequencing (for the EP300 gene), we studied here 13 patients who had shown no mutation after genomic sequencing of the CREBBP gene in a previous investigation. Two new disease-causing mutations were identified, including a partial deletion of CREBBP and a 1-bp deletion in EP300, c.7100delC (p.P2366fsX2401). The 1-bp deletion represents the fourth EP300 mutation reported to date and was identified in a patient with non-classical RSTS. Based on the very similar structure of the CREBBP and EP300 genes and the higher rate of single-nucleotide polymorphisms in EP300 (2.23 per individual) as compared to CREBBP (0.71 per individual) $(P>0.001$, Wilcoxon test), it may be assumed that EP300 gene mutations should be as frequent as CREBBP gene mutations. Based on the location of the EP300 gene mutations identified so far (outside the histone acetyl transferase domain) and the observed (although not very striking) phenotypical differences with the EP300 mutations, we propose that most EP300 mutations could be associated with other phenotypes, not classical RSTS.

European Journal of Human Genetics (2007) 15, 837-842; doi:10.1038/sj.ejhg.5201791; published online 14 February 2007

Keywords: Rubinstein-Taybi syndrome; CREBBP; EP300; frameshift mutation; histone acetyl transferase domain

\section{Introduction}

Rubinstein-Taybi syndrome (RSTS) is a well-known autosomal-dominant disorder of typical face, short stature, skeletal abnormalities, and mental retardation, and was shown to be caused in most cases by mutations in CREBBP, the gene for CREB binding protein. ${ }^{1-3}$ Approximately $45-$ $56 \%$ of patients with RSTS demonstrated CREBBP mutations, ${ }^{2,3}$ and recently, three individuals with EP300 gene

*Correspondence: Priv-Doz Dr O Bartsch, Institut für Humangenetik, Universitätsklinikum, Langenbeckstr. 1, D-55101 Mainz, Germany.

Tel: + 496131 175791; Fax: + 496131 175690;

E-mail: bartsch@humgen.klinik.uni-mainz.de

Received 6 November 2006; revised 20 December 2006; accepted 10 January 2007; published online 14 February 2007 mutations were reported in a series of 92 patients. ${ }^{4}$ The EP300 gene on chromosome 22q13 encodes a protein, p300, that is highly similar to CREBBP. Both CREBBP and p300 are transcriptional coactivators with a plant homeodomain-type zinc finger and a histone acetyl transferase (HAT) domain facilitating chromatin opening. ${ }^{5,6}$ They are involved in the regulation of the expression of numerous genes that are important in embryonic development, cell growth, cellular differentiation, and tumor suppression. ${ }^{7-10}$ Despite many similarities, CREBBP and p300 are involved in slightly different signal-transduction pathways. ${ }^{7,9-12}$ The first report of EP300 mutations in RSTS $^{4}$ required a confirmational study (performed here) and raised new questions, for example, regarding the frequency of 
EP300 mutations in RSTS, the causes of the paucity of EP300 vs CREBBP mutations, the phenotypic spectrum in humans with EP300 mutations, and possible further genetic heterogeneity in RSTS.

Using genomic sequencing and real-time quantitative PCR (qPCR) we studied 13 patients (out of a series of 38 patients previously reported after fluorescence in situ hybridization (FISH) and genomic sequencing of CREBBP), ${ }^{3}$ for the presence of EP300 mutations and CREBBP deletions that had escaped detection by FISH. We identified two novel mutations, a 1-bp deletion (c.7100delC) in EP3OO and a CREBBP deletion including exons 28 and 31 . The $E P 300$ gene mutation predicts a very mildly truncated protein and does not alter the HAT domain, which elsewhere had been shown to be critical in causing RSTS. ${ }^{5,6}$ Based on the location of the EP300 gene mutations $^{4}$ (also this study), the relatively large number of single-nucleotide polymorphisms (SNPs; 2.33 per patient), and the observed (although not very striking) phenotypical differences with the EP300 mutations detected so far $^{4}$ (also this study), we suggest that mutations in EP300 could be underdiagnosed because they may result in phenotypes different from RSTS. Finally, we identified in EP300 a homozygous sequence variation $(c .2053+8 \mathrm{~T})$ that was present in all individuals studied and therefore most likely represents the wild-type sequence.

\section{Materials and methods}

Out of a series of 38 previously reported patients, we studied here 13 of 19 patients in whom no CREBBP mutation had been identified. ${ }^{3}$ Of these, 10 individuals had RSTS and three had a diagnosis of incomplete RSTS (see $^{3}$ for definition). From the other six patients without detectable CREBBP mutation, there was not enough DNA at disposal.

We used genomic sequencing and real-time $\mathrm{qPCR}^{13,14}$ for the detection of molecular EP300 mutations and partial deletions of CREBBP and EP300, respectively. The 31 coding exons of EP300 were analyzed in 37 fragments (Table 1) using a previously described protocol ${ }^{3,15}$ and a $\mathrm{CEQ}^{\mathrm{TM}} 8000$ Genetic Analyzer (Beckman Coulter, Fullerton, CA, USA). All sequence variations were confirmed in a second PCR and sequencing run. The EP300 gene mutation identified here was proven to be de novo by sequencing of DNA from the parents. Paternity testing was not performed due to legal restrictions. The Entrez SNP Database was consulted for interpretation of SNPs. The qPCR was performed using a total of 18 different amplicons (CREBBP: intron 1 , exon 2 , introns $2-3$, exon 3 , introns $4-5$, exon 6 , exon 14, exon 16, exon 28, and exon 31; EP300: exons 1, 2, 4 , intron 9, and exons $14,18,25$, and 31 ) and a previously described protocol. ${ }^{13}$
Note that $C R E B B P$ and EP3OO exon numbers are in line with the literature, ${ }^{1-6}$ but differ by -1 from actual exon numbers.

\section{Results}

We identified two mutations. In patient 12 described below, genomic sequencing of EP300 revealed a 1-bp deletion, c.7100delC (p.P2366fsX2401; Figure 1). In patient 21, a 21-year-old woman with RSTS (A-1928-22 in our previous study), ${ }^{3}$ qPCR indicated a deletion of CREBBP at exons 28 and 31. Thus, the CREBBP mutation detection rate increased to $52.6 \%$ (20 in 38) in our whole series of patients, or to $60 \%$ (18 in 30) in the subset of patients with unequivocal RSTS only.

We also detected five different SNPs in the EP300 gene (Table 2), all previously reported, including non-synonymous SNPs in exons 3 (c.865A $\rightarrow$ G, p.M289V, rs2230111) and 15 (c.2989A $\rightarrow \mathrm{G}$, p.I997V, rs20551), a synonymous SNP in exon 17 (c.3183A $\rightarrow$ T, rs4822012), and two intronic variants (introns $27-28$, c. $4452+20 \mathrm{C} \rightarrow \mathrm{T}$, rs6002271; introns 28-29, c.4618-18C $\rightarrow \mathrm{T}$, rs2076578). Moreover, all patients in this study and two normal controls demonstrated the same homozygous sequence variation at introns 10-11 (c.2053 + 8G $\rightarrow$ T, rs6002267) (Figure 1).

Patient 12 (B-3444-13 in our previous study) $)^{3}$ showing the EP300 gene mutation was first diagnosed with 'mild variant but unquestionably' RSTS by the late Professor Frank Majewski, Universitätsklinikum Düsseldorf, Germany, who assessed in 2001 that '... of all patients with RSTS whom I have seen (indeed many), she is by far the best as regards intelligence. All others were mentally retarded, but this is certainly not true for this patient. Therefore one would ... rather expect an atypical mutation.' [... Von allen Patienten mit RTS, die ich bisher gesehen habe (wirklich zahlreich!), ist sie mit Abstand die beste, was die Intelligenz betrifft. Alle anderen waren geistig behindert, was bei dieser Patientin sicher nicht zutrifft. Insofern ist ... eher mit einer atypischen Mutation zu rechnen.] At the age of 14 years and 3 months she presented with microcephaly (occipitofrontal circumference $49.5 \mathrm{~cm},-3.2 \mathrm{SD}$ ) and a very beaked nose, columella below alae nasi, narrow high-arched palate, a very pronounced overbite with the maxillary incisors markedly protruding over the lower lip, and retrognathia. She had mild myopia $(-1 \mathrm{D})$ and broad thumbs and big toes. Height $(152.8 \mathrm{~cm},-1.4 \mathrm{SD})$ was normal and intelligence was borderline normal. At age 18 years, the pronounced overbite was corrected by maxillary surgery. She attended an integrated school for the learning disabled, but after 10 classes failed in the final examination. Vocational training was viewless and she now works in a workshop for the mentally handicapped (estimated IQ $\sim 75)$. The family provided full-length portraits to one of us (OB) but denied permission to publication. The photos 
Table 1 Primers and PCR conditions for EP300 sequencing

\begin{tabular}{|c|c|c|c|c|}
\hline EP300 exon (size) & Position $^{\mathrm{a}}$ & Primer name (fragment size) & Primer sequence $5^{\prime} \rightarrow 3^{\prime}$ & Annealing temperature \\
\hline Exon 1 (94 bp) & $190,+151$ & $\begin{array}{l}\text { Ex01-F (435 bp) } \\
\text { Ex01-R }\end{array}$ & $\begin{array}{l}\text { TITCTATCGAGTCCGCATCC } \\
\text { ACGTCTTCGACCAGCTCATT }\end{array}$ & $53^{\circ} \mathrm{C}$ \\
\hline Exon 2 (635 bp) & -108 & Ex02-F1 (536 bp) & GGAGTGAGGTTGGGAAATGA & $58.9^{\circ} \mathrm{C}$ \\
\hline & $428^{\mathrm{b}}$ & Ex02-R1 & CAGCCAACATTCCAGGATTC & \\
\hline Exon 3 (177bp) & $-197,+106$ & Ex03-F (480 bp) & GCCACCATGTCCAGATTITT & $55.5^{\circ} \mathrm{C}$ \\
\hline Exon $4(262 b p)$ & $-141,+50$ & $\begin{array}{l}\text { Ex04-F (453 bp) } \\
\text { Ex04-R }\end{array}$ & $\begin{array}{l}\text { TGCATTCCCTGTGTCAAAAA } \\
\text { TCCCTGGCTGTAAAAATTGC }\end{array}$ & $52.3^{\circ} \mathrm{C}$ \\
\hline Exon 5 (114bp) & $-200,+98$ & $\begin{array}{l}\text { Ex05-F (412 bp) } \\
\text { Ex05-R }\end{array}$ & $\begin{array}{l}\text { TTTGTGCAAATTGCTTACCC } \\
\text { ACACCACAGGTCCCTCACAT }\end{array}$ & $55.5^{\circ} \mathrm{C}$ \\
\hline Exon 6 (246 bp) & $-187,+49$ & $\begin{array}{l}\text { Ex06-F (482 bp) } \\
\text { Ex06-R }\end{array}$ & $\begin{array}{l}\text { CATTTACTGACACAACCAATACCA } \\
\text { TGGTCCCCTTTACCAATCAG }\end{array}$ & $55.5^{\circ} \mathrm{C}$ \\
\hline Exon 7 (94 bp) & $-263,+103$ & $\begin{array}{l}\text { Ex07-F (460 bp) } \\
\text { Ex07-R }\end{array}$ & $\begin{array}{l}\text { GCCTGGTCACATTTGCTTTT } \\
\text { GACATCCTCAAACCGAGGAA }\end{array}$ & $52.3^{\circ} \mathrm{C}$ \\
\hline Exon 8 (138bp ) & $-145,+157$ & $\begin{array}{l}\text { Ex08-F (440 bp) } \\
\text { Ex08-R }\end{array}$ & $\begin{array}{l}\text { CACACTTCTCCCTGCCTAGC } \\
\text { CAGCAGCAAGAAATCCACAA }\end{array}$ & $55.5^{\circ} \mathrm{C}$ \\
\hline Exon 9 (118bp) & $-298,+50$ & $\begin{array}{l}\text { Ex09-F (466 bp) } \\
\text { Ex09-R }\end{array}$ & $\begin{array}{l}\text { TGACAAGGCCTGTTTTCCTC } \\
\text { CCACAACAGGTTCAATCTTGG }\end{array}$ & $55.5^{\circ} \mathrm{C}$ \\
\hline Exon 10 (175 bp) & $-126,+130$ & $\begin{array}{l}\text { Ex10-F (431 bp) } \\
\text { Ex10-R }\end{array}$ & $\begin{array}{l}\text { TTGGCACCAGTTCTTAATGC } \\
\text { TTTGCAAAGGCAAATGGTTA }\end{array}$ & $52^{\circ} \mathrm{C}$ \\
\hline Exon 11 (78 bp) & $-200,+173$ & $\begin{array}{l}\text { Ex11-F (451 bp) } \\
\text { Ex11-R }\end{array}$ & $\begin{array}{l}\text { TCCACTTGGAGGCATTITC } \\
\text { AATGTCACCTGCCCTGTGAT }\end{array}$ & $55.5^{\circ} \mathrm{C}$ \\
\hline Exon 12 (110 bp) & $-189,+200$ & $\begin{array}{l}\text { Ex12-F (499bp) } \\
\text { Ex12-R }\end{array}$ & $\begin{array}{l}\text { GAATGGTGTGAACCCAGGTG } \\
\text { CACTGACACTCCAGGGACAA }\end{array}$ & $55.5^{\circ} \mathrm{C}$ \\
\hline Exon 13 (138bp) & $-184,+95$ & $\begin{array}{l}\text { Ex13-F (417bp) } \\
\text { Ex13-R }\end{array}$ & $\begin{array}{l}\text { TGGCACAAGAGTCAGTTGTTCT } \\
\text { ACCCACTATTTGCTGCСACT }\end{array}$ & $53^{\circ} \mathrm{C}$ \\
\hline Exon 14 (791 bp) & $-180,+58$ & $\begin{array}{l}\text { Ex14-F (553 bp) } \\
\text { Ex14-R }\end{array}$ & $\begin{array}{l}\text { CTTGATGGTGCTGTCCAAAG } \\
\text { GAATGGAAATGGCCCAGAAG }\end{array}$ & $57^{\circ} \mathrm{C}$ \\
\hline Exon 15 (180 bp) & $-154,+132$ & $\begin{array}{l}\text { Ex15-F (466 bp) } \\
\text { Ex15-R }\end{array}$ & $\begin{array}{l}\text { GTGAAATGGGCAGAGCAAAT } \\
\text { GAACACCСTTTCTCATGCAA }\end{array}$ & $52.3^{\circ} \mathrm{C}$ \\
\hline Exon 16 (145 bp) & $-214,+127$ & $\begin{array}{l}\text { Ex16-F (486 bp) } \\
\text { Ex16-R }\end{array}$ & $\begin{array}{l}\text { CAGCCGGAGGATATTTCAGA } \\
\text { CAGGGCTTCCAGGTTAATGA }\end{array}$ & $55.5^{\circ} \mathrm{C}$ \\
\hline Exon 17 (119bp) & $-146,+72$ & $\begin{array}{l}\text { Ex17-F (337 bp) } \\
\text { Ex17-R }\end{array}$ & $\begin{array}{l}\text { GAGCCTGTAGTGATATTTCC } \\
\text { GAGTGGCTATACTCTTTGG }\end{array}$ & $55^{\circ} \mathrm{C}$ \\
\hline Exon 18 (240 bp) & $-138,+105$ & $\begin{array}{l}\text { Ex18-F (483 bp) } \\
\text { Ex18-R }\end{array}$ & $\begin{array}{l}\text { TCAGTCACCTCTTGGGGAATA } \\
\text { AGCCATGTCAAAGCCAGAAG }\end{array}$ & $53.1^{\circ} \mathrm{C}$ \\
\hline Exon 19 (89 bp) & $-190,+205$ & $\begin{array}{l}\text { Ex19-F (484 bp) } \\
\text { Ex19-R }\end{array}$ & $\begin{array}{l}\text { AGCCTACCTCAGCGTTTGA } \\
\text { CCCAGGTAACTGATGCAAGG }\end{array}$ & $55.5^{\circ} \mathrm{C}$ \\
\hline Exon 20 (147bp) & $-94,+177$ & $\begin{array}{l}\text { Ex20-F (418bp) } \\
\text { Ex20-R }\end{array}$ & $\begin{array}{l}\text { CGGAACAGTTCACCCCAGTA } \\
\text { GCCCATTGCTGACATATTCC }\end{array}$ & $53^{\circ} \mathrm{C}$ \\
\hline Exon 21 (81 bp) & $-38,+301$ & $\begin{array}{l}\text { Ex21-F (420 bp) } \\
\text { Ex21-R }\end{array}$ & $\begin{array}{l}\text { GGGTGAAGTTTGTTCCTTTGG } \\
\text { TTCCTGGTGCTGAAAATTCC }\end{array}$ & $55.5^{\circ} \mathrm{C}$ \\
\hline Exon 22 (57 bp) & $-87,+348$ & $\begin{array}{l}\text { Ex22-F (492 bp) } \\
\text { Ex22-R }\end{array}$ & $\begin{array}{l}\text { TTGGAATTGGCTCTGCTCTT } \\
\text { ACCTCGCCTGGCCATAAATA }\end{array}$ & $55.5^{\circ} \mathrm{C}$ \\
\hline Exon 23 (78 bp) & $-24,+312$ & $\begin{array}{l}\text { Ex23-F (414bp) } \\
\text { Ex23-R }\end{array}$ & $\begin{array}{l}\text { ATGCCСTTCATGTTCTTCA } \\
\text { TGCATTCTACAAATCCGTTCC }\end{array}$ & $55.5^{\circ} \mathrm{C}$ \\
\hline Exon 24 (68 bp) & $-216,+215$ & $\begin{array}{l}\text { Ex24-F (499bp) } \\
\text { Ex24-R }\end{array}$ & $\begin{array}{l}\text { TTAGCATGTTCCCTGCACTC } \\
\text { CTGCСАTCTCTCСАСТGTCC }\end{array}$ & $51.5^{\circ} \mathrm{C}$ \\
\hline Exon 25 (151 bp) & $-189,+147$ & $\begin{array}{l}\text { Ex25-F (487 bp) } \\
\text { Ex25-R }\end{array}$ & $\begin{array}{l}\text { CTCGTGGATCCAAAATTGCT } \\
\text { TATTITGTITGGGGGTITGC }\end{array}$ & $55.5^{\circ} \mathrm{C}$ \\
\hline Exon 26 (114bp) & $-199,+151$ & $\begin{array}{l}\text { Ex26-F (464 bp) } \\
\text { Ex26-R }\end{array}$ & $\begin{array}{l}\text { AGAAGGAAACCACAGGCTCA } \\
\text { AAAGGGGCTCCAACAAAGTT }\end{array}$ & $56.2^{\circ} \mathrm{C}$ \\
\hline Exon 27 (166 bp) & $-168,+164$ & $\begin{array}{l}\text { Ex27-F (498 bp) } \\
\text { Ex27-R }\end{array}$ & $\begin{array}{l}\text { GCCTAATTTTGCCTCACAA } \\
\text { GAGCCAAAATCGTGTCACTG }\end{array}$ & $56.2^{\circ} \mathrm{C}$ \\
\hline Exon 28 (165 bp) & $-116,+200$ & $\begin{array}{l}\text { Ex28-F (481 bp) } \\
\text { Ex28-R }\end{array}$ & $\begin{array}{l}\text { TGCCAGCTTTCAAGACATTTT } \\
\text { AAAAGGGGCTCTGATGCTTT }\end{array}$ & $53^{\circ} \mathrm{C}$ \\
\hline Exon 29 (162 bp) & $-285,+48$ & $\begin{array}{l}\text { Ex29-F (495 bp) } \\
\text { Ex29-R }\end{array}$ & $\begin{array}{l}\text { TAGCCCCAATCTGGGATACA } \\
\text { GCCAGAAATCTTGCCGTIT }\end{array}$ & $53^{\circ} \mathrm{C}$ \\
\hline Exon 30 (282 bp) & $-104,+76$ & $\begin{array}{l}\text { Ex30-F (462 bp) } \\
\text { Ex30-R }\end{array}$ & $\begin{array}{l}\text { CCAGGAGGCAGAGGTTGTAG } \\
\text { AGCATCCCACAGGCCTCTAT }\end{array}$ & $59^{\circ} \mathrm{C}$ \\
\hline Exon 31 (2184 bp) & $\begin{array}{l}-95 \\
322^{\mathrm{b}} \\
247^{\mathrm{b}} \\
747^{\mathrm{b}}\end{array}$ & $\begin{array}{l}\text { Ex31-F1 (417bp) } \\
\text { Ex31-R1 } \\
\text { Ex31-F2 (500 bp) } \\
\text { Ex31-R2 }\end{array}$ & $\begin{array}{l}\text { ACGAAAGGGGCTTTCTAGC } \\
\text { GCATITGTTCTCCTGGCAGT } \\
\text { GTTGCAAACGGAAAACCAAT } \\
\text { CGCTGCTCTCTGAATCTGC }\end{array}$ & $\begin{array}{l}54.7^{\circ} \mathrm{C} \\
54.7^{\circ} \mathrm{C}\end{array}$ \\
\hline
\end{tabular}


Table 1 (Continued)

\begin{tabular}{|c|c|c|c|c|}
\hline EP300 exon (size) & Position $^{a}$ & Primer name (fragment size) & Primer sequence $5^{\prime} \rightarrow 3^{\prime}$ & Annealing temperature \\
\hline & $\begin{array}{l}658^{\mathrm{b}} \\
1129^{\mathrm{b}} \\
1032^{\mathrm{b}} \\
1522^{\mathrm{b}} \\
1474^{\mathrm{b}} \\
1961^{\mathrm{b}} \\
1869^{\mathrm{b}} \\
+120\end{array}$ & $\begin{array}{l}\text { Ex31-F3 (471 bp) } \\
\text { Ex31-R3 } \\
\text { Ex31-F4 (490 bp) } \\
\text { Ex31-R4 } \\
\text { Ex31-F5 (487 bp) } \\
\text { Ex31-R5 } \\
\text { Ex31-F6 (435 bp) } \\
\text { Ex31-R6 }\end{array}$ & $\begin{array}{l}\text { CCCTCAGGTTCATCTTGCAT } \\
\text { GAGAGCTGGGAGACCTGAGA } \\
\text { GGATTGGGCCAGGTAGGTAT } \\
\text { GTTGCTGCTGCTGTTGCAT } \\
\text { TCCGAGACATCTTGAGACGA } \\
\text { GAAACGTGGTGTGGAGAAGG } \\
\text { CTTCTCCACGGCCACAGT } \\
\text { ACGGCATACTGCACAGTTCTT }\end{array}$ & $\begin{array}{l}55.5^{\circ} \mathrm{C} \\
54.7^{\circ} \mathrm{C} \\
54.7^{\circ} \mathrm{C} \\
57.5^{\circ} \mathrm{C}\end{array}$ \\
\hline
\end{tabular}

aPosition of left and right primers relative to first and last nucleotide of exon, respectively.

bosition within exon, given relative to first nucleotide of exon 31.

a

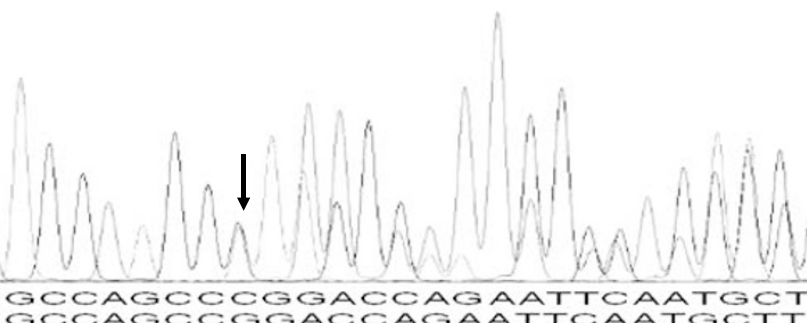

GOOAGOTOTCAATGOT

b

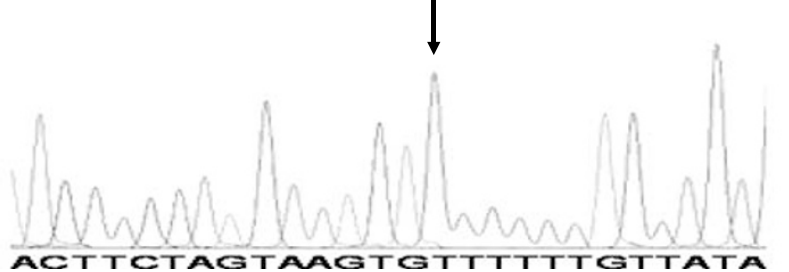

C

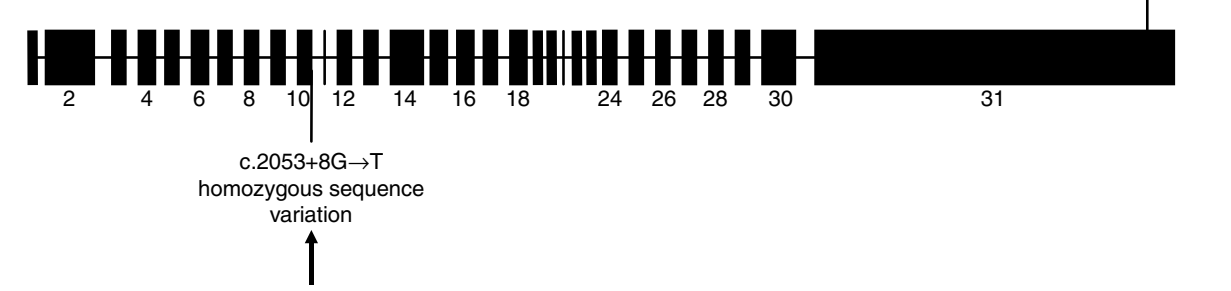

Figure 1 EP300 gene, (a) the 1-bp deletion, c.7100delC, identified in patient 12 with mild variant RSTS and borderline intelligence. (b) The thymine residue at position 8 of the exon 10 splice donor (c.2053 + 8T), which was previously reported as a SNP (c.2053 + 8G $\rightarrow$ T, rs6002267), but identified in homozygous state in all study patients and two healthy controls, suggesting that thymine is the wild-type sequence. (c) Position of the c.7100delC (p.P2366fsX2401) mutation and of the c.2053+8T 'SNP' within the EP300 gene.

showed no classical RSTS, as also evident from the pronounced overbite and orthodontic surgery performed here.

\section{Discussion}

The c.7100delC deletion in exon 31 represents the fourth disease-associated mutation in EP30O reported to date and the first confirmation of the results of Roelfsema et $a l^{4}$ It predicts a premature stop codon, p.P2366fsX2401, and a protein truncation towards the $3^{\prime}$-end (normal size, 2414 residues). Roelfsema et $\mathrm{al}^{4}$ described two EP300 mutations (one nonsense, one frameshift) predicting small truncated proteins without the HAT domain, and a third mutation probably deleting essential parts of the EP300 promotor region and probably not resulting in any protein at all. The c.7100delC deletion occurred de novo and in all likelihood represents the causative mutation in our patient, not a polymorphism. Frameshift and nonsense mutations affecting the HAT domain represent the most frequent mutation types observed in RSTS with the CREBBP $P^{1-3,5,6}$ and $E P 300^{4}$ genes. However, the location of the present frameshift 
Table 2 Twenty-nine single-nucleotide polymorphisms of the EP300 gene, including three homozygous SNPs, detected in 13 patients

\begin{tabular}{|c|c|c|c|c|c|}
\hline Patient $^{\mathrm{a}}$ & Internal code ${ }^{a}$ & Location & Mutation & & $d b S N P$ rs\# ${ }^{\mathrm{b}}$ \\
\hline 24 & B-3510-25 & Exon 3 & c. $865 \mathrm{~A} \rightarrow \mathrm{G}$ & Non synonymous, p.M289V & 2230111 \\
\hline 8 & A-3435-09 & Exon 15 & c. $2989 A \rightarrow G$ & Non synonymous, p.1997V & 20551 \\
\hline 12 & B-3444-13 & Exon 15 & C. $2989 \mathrm{~A} \rightarrow \mathrm{G}^{\mathrm{C}}$ & Non synonymous, p.1997V & 20551 \\
\hline 13 & B-3446-14 & Exon 15 & c. $2989 A \rightarrow G$ & Non synonymous, p.1997V & 20551 \\
\hline 18 & A-3478-19 & Exon 15 & c. $2989 A \rightarrow G$ & Non synonymous, p.1997V & 20551 \\
\hline 33 & A-3584-35 & Exon 15 & c. $2989 A \rightarrow G$ & Non synonymous, p.1997V & 20551 \\
\hline 10 & A-3437-11 & Exon 17 & c. $3183 \mathrm{~A} \rightarrow \mathrm{T}$ & Synonymous & 4822012 \\
\hline 13 & B-3446-14 & Exon 17 & c. $3183 \mathrm{~A} \rightarrow \mathrm{T}$ & Synonymous & 4822012 \\
\hline 15 & A-3463-16 & Exon 17 & c. $3183 \mathrm{~A} \rightarrow \mathrm{T}$ & Synonymous & 4822012 \\
\hline 20 & A-1878-21 & Exon 17 & c. $3183 \mathrm{~A} \rightarrow \mathrm{T}$ & Synonymous & 4822012 \\
\hline 24 & $B-3510-25$ & Exon 17 & c. $3183 \mathrm{~A} \rightarrow \mathrm{T}$ & Synonymous & 4822012 \\
\hline 33 & $A-3584-35$ & Exon 17 & c. $3183 \mathrm{~A} \rightarrow \mathrm{T}$ & Synonymous & 4822012 \\
\hline 44 & $A-3639-47$ & Exon 17 & c. $3183 \mathrm{~A} \rightarrow \mathrm{T}$ & Synonymous & 4822012 \\
\hline 8 & A-3435-09 & Exon $27-28$ & c. $4452+20 \mathrm{C} \rightarrow \mathrm{T}$ & Intron & 6002271 \\
\hline 12 & B-3444-13 & Exon $27-28$ & c. $4452+20 \mathrm{C} \rightarrow \mathrm{T}$ & Intron & 6002271 \\
\hline 13 & B-3446-14 & Exon $27-28$ & c. $4452+20 \mathrm{C} \rightarrow \mathrm{T}$ & Intron & 6002271 \\
\hline 18 & A-3478-19 & Exon $27-28$ & c. $4452+20 \mathrm{C} \rightarrow \mathrm{T}$ & Intron & 6002271 \\
\hline 33 & $A-3584-35$ & Exon $27-28$ & $\mathrm{c} .4452+20 \mathrm{C} \rightarrow \mathrm{T}$ & Intron & 6002271 \\
\hline 7 & A-3433-08 & Exon $28-29$ & c. $4618-18 \mathrm{C} \rightarrow \mathrm{T}^{\mathrm{C}}$ & Intron & 2076578 \\
\hline 10 & A-3437-11 & Exon $28-29$ & c. $4618-18 \mathrm{C} \rightarrow \mathrm{T}$ & Intron & 2076578 \\
\hline 15 & A-3463-16 & Exon $28-29$ & c. $4618-18 \mathrm{C} \rightarrow \mathrm{T}$ & Intron & 2076578 \\
\hline 20 & A-1878-21 & Exon 28-29 & c. $4618-18 \mathrm{C} \rightarrow \mathrm{T}$ & Intron & 2076578 \\
\hline 21 & A-1928-22 & Exon $28-29$ & c. $4618-18 \mathrm{C} \rightarrow \mathrm{T}$ & Intron & 2076578 \\
\hline 24 & B-3510-25 & Exon 28-29 & c. $4618-18 \mathrm{C} \rightarrow \mathrm{T}$ & Intron & 2076578 \\
\hline 27 & $A-3508-28$ & Exon $28-29$ & $\mathrm{c} .4618-18 \mathrm{C} \rightarrow \mathrm{T}^{\mathrm{C}}$ & Intron & 2076578 \\
\hline 44 & A-3639-47 & Exon $28-29$ & c. $4618-18 \mathrm{C} \rightarrow \mathrm{T}$ & Intron & 2076578 \\
\hline
\end{tabular}

${ }^{\mathrm{a}}$ Patient and code numbers same as in previous publication; Bartsch et al. ${ }^{3}$ letters $\mathrm{A}$ or $\mathrm{B}$ in internal code indicate phenotype: $\mathrm{A}=\mathrm{RSTS}, \mathrm{B}=$ incomplete RSTS.

${ }^{\mathrm{b}}$ Polymorphism previously reported in the Entrez Single-Nucleotide Polymorphism Database (dbSNP).

${ }^{\mathrm{C}}$ Homozygous SNP.

mutation very close to the $3^{\prime}$-end of EP300 is interesting; ${ }^{3,5,6,11,16}$ it leads to a putative very mild truncation that does not alter the HAT domain, and could possibly explain the non-classical RSTS (unusual face, relatively good intelligence) in our patient. An overbite with the maxillary incisors markedly protruding over the lower lip and requiring maxillary surgery is an unusual feature in RSTS. $^{17}$

We identified only one EP300 mutation in this series of 38 patients, or $\sim 2.6 \%$, a frequency corresponding well with the previous report of $\sim 3.2 \%$ EP300 mutations. ${ }^{4}$ Considering the similar genetic properties and functions of CREBBP (31 exons, 2442 residues) and EP300 (31 exons, 2414 residues), one might expect similar rates of mutations and similar sites of pathogenic mutations. Hence, the 20 -fold difference (52.6 vs 2.6\%) between the rates of detectable mutations in CREBBP and EP3OO in our patients strongly suggests that EP3OO plays only a minor role in the etiology of RSTS. The relatively low total number of mutations identified in CREBBP and EP3OO (60\%, considering the most stringent diagnostic group A) could be in support of further genetic heterogeneity in RSTS.

Furthermore we detected in the EP300 gene five SNPs (Table 2; two non-synonymous SNPs, a synonymous SNP and two intronic variants). Addressing the lower rate of pathogenic mutations in EP300 as compared to CREBBP, Roelfsema et $a l^{4}$ discussed the possibility of different chances of mutations occurring in these two genes, despite their very similar genetic composition (31 coding exons, $\sim 2400$ residues). Alternatively, mutation rates could be similar and the vast majority of individuals with EP300 mutations could demonstrate phenotypes other than RSTS. In our previous study, ${ }^{3}$ we identified 15 different SNPs in CREBBP in 38 patients ( 0.39 per individual) and here we detected five different EP300 SNPs in 13 patients ( 0.38 per individual), a finding supporting approximately equal mutation rates in the two genes. However, the 13 patients in this study demonstrated a total of 29 SNPs in EP300 (Table 2), or 2.23 per individual, as compared to 27 CREBBP polymorphisms in 38 patients, or 0.71 per individual, identified in our previous study. ${ }^{3}$ Hence, EP300 polymorphisms were present at higher frequencies (Wilcoxon test, $\mathrm{p}<0.001)$. The higher frequencies of the EP300 SNPs could possibly reflect differences in genetic drift of the different polymorphisms. Taken together, our data support that EP300 mutations should be roughly about as frequent as CREBBP mutations, but some could lead to other phenotypes, not classical RSTS. 
Similarly, the very different frequencies of CREBBP and EP300 mutations with the RSTS phenotype and the observed (although not very striking) phenotypical differences with the EP300 mutations ${ }^{4}$ (also this study) suggest that mutations in EP300 could be underdiagnosed due to phenotypes different from classical RSTS. A possible explanation could be the different functions of CREBBP and EP300 during embryogenesis. 7,9,18

Finally, a homozygous sequence variation (EP300 c. $2053+8 \mathrm{~T})$ was present in all individuals studied and hence most likely represents the wild-type sequence. We therefore suggest that the guanine at position 8 of the exon 10 splice donor in the current version of the human genome reference sequence represents a polymorphism.

\section{Acknowledgements}

We thank the patients and their families for contributing to this study; our clinical colleagues for referring patients and clinical data, especially Professor Frank Majewski (University of Düsseldorf, Germany; patient 12), and Cornelia Wetzel and Stanislav Lekhno for assistance in the laboratory.

\section{Electronic-database information}

URLs and accession numbers for data in this article are as follows: Online Mendelian Inheritance in Man (OMIM, http:// www.ncbi.nlm.nih.gov/Omim) for the Rubinstein-Taybi syndrome (MIM 180849). Ensembl (http://www.ensembl.org/ Homo_sapiens/index.html) for CREBBP and EP300 sequences. Single-Nucleotide Polymorphism Database (dbSNP, http:// www.ncbi.nlm.nih.gov/SNP/) for SNPs in EP300 and CREBBP. Primer 3 Output software (http://www.genome.wi.mit.edu// cgi-bin/primer/primer3_www.cgi).

\section{References}

1 Petrij F, Giles RH, Dauwerse HG et al: Rubinstein-Taybi syndrome caused by mutations in the transcriptional co-activator CBP. Nature 1995; 376: 348-351.

2 Coupry I, Roudaut C, Stef M et al: Molecular analysis of the CBP gene in 60 patients with Rubinstein-Taybi syndrome. J Med Genet 2002; 39: 415-421.
3 Bartsch O, Schmidt S, Richter M et al: DNA sequencing of CREBBP demonstrates mutations in $56 \%$ of patients with RubinsteinTaybi syndrome (RSTS) and in another patient with incomplete RSTS. Hum Genet 2005; 117: 485-493.

4 Roelfsema JH, White SJ, Ariyurek Y et al: Genetic heterogeneity in Rubinstein-Taybi syndrome: mutations in both the CBP and EP300 genes cause disease. Am J Hum Genet 2005; 76: 572-580.

5 Murata T, Kurokawa R, Krones A et al: Defect of histone acetyltransferase activity of the nuclear transcriptional coactivator CBP in Rubinstein-Taybi syndrome. Hum Mol Genet 2001; 10: 1071-1076.

6 Kalkhoven E, Roelfsema JH, Teunissen $\mathrm{H}$ et al: Loss of CBP acetyltransferase activity by PHD finger mutations in Rubinstein-Taybi syndrome. Hum Mol Genet 2003; 12: 441-450.

7 Giles RH, Peters DJ, Breuning MH: Conjunction dysfunction: CBP/p300 in human disease. Trends Genet 1998; 14: 178-183.

8 Yao TP, Oh SP, Fuchs M et al: Gene dosage-dependent embryonic development and proliferation defects in mice lacking the transcriptional integrator p300. Cell 1998; 93: 361-372.

9 Partanen A, Motoyama J, Hui CC: Developmentally regulated expression of the transcriptional cofactors/histone acetyltransferases CBP and p300 during mouse embryogenesis. Int J Dev Biol 1999; 43: 487-494.

10 Goodman RH, Smolik S: CBP/p300 in cell growth, transformation, and development. Genes Dev 2000; 14: 1553-1578.

11 Roth JF, Shikama N, Henzen C et al: Differential role of p300 and CBP acetyltransferase during myogenesis: p300 acts upstream of MyoD and Myf5. EMBO J 2003; 22: 5186-5196.

12 Kalkhoven E: CBP and p300: HATs for different occasions. Biochem Pharmacol 2004; 68: 1145-1155.

13 Borozdin W, Boehm D, Leipoldt M et al: SALL4 deletions are a common cause of Okihiro and acro-renal-ocular syndromes and confirm haploinsufficiency as the pathogenetic mechanism. J Med Genet 2004; 41: e113.

14 Coupry I, Monnet L, Attia AA, Taine L, Lacombe D, Arveiler B: Analysis of CBP CREBBP) gene deletions in Rubinstein-Taybi syndrome patients using real-time quantitative PCR. Hum Mutat 2004; 23: 278-284.

15 Bartsch O, Locher K, Meinecke P et al: Molecular studies in 10 cases of Rubinstein-Taybi syndrome, including a mild variant showing a missense mutation in codon 1175 of CREBBP. J Med Genet 2002; 39: 496-501.

16 Ugai H, Uchida K, Kawasaki H, Yokoyama KK: The coactivators p300 and CBP have different functions during the differentiation of F9 cells. J Mol Med 1999; 77: 481-494.

17 Hennekam RCM: Rubinstein-Taybi syndrome. Eur J Hum Genet 2006; 14: 981-985.

18 Kawasaki H, Eckner R, Yao TP et al: Distinct roles of the coactivators p300 and CBP in retinoic-acid-induced F9-cell differentiation. Nature 1998; 393: 285-289. 